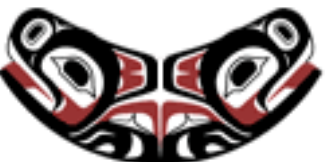

UW Biostatistics Working Paper Series

$12-19-2003$

\title{
Survival Model Predictive Accuracy and ROC Curves
}

Patrick Heagerty

University of Washington, heagerty@u.washington.edu

Yingye Zheng

Fred Hutchinson Cancer Research Center, yzheng@fhcrc.org

\section{Suggested Citation}

Heagerty, Patrick and Zheng, Yingye, "Survival Model Predictive Accuracy and ROC Curves" (December 2003). UW Biostatistics Working Paper Series. Working Paper 219.

http://biostats.bepress.com/uwbiostat/paper219

This working paper is hosted by The Berkeley Electronic Press (bepress) and may not be commercially reproduced without the permission of the copyright holder.

Copyright $(\odot 2011$ by the authors 


\section{Introduction}

In this manuscript we propose a new method for characterizing the predictive accuracy of a regression model when the outcome of interest is a censored survival time. We focus on data obtained from a prospective study in which a continuous follow-up time is observed for each participant, but follow-up can be terminated either by the occurance of the event of interest or by censoring. Thus the essential outcome information is the combination of the status at the end of follow-up (binary) and the length of follow-up (continuous). Since censored data share features of both continuous response data and binary data the accuracy concepts that are standard for either response type may be extended for survival outcomes. Previous research has focused on extending the proportion of variation explained by the covariates, or $R^{2}$, to censored data models (Schemper and Henderson 2000; O'Quigley and Xu 2001). In addition, limited work has explored the use of familiar binary outcome methods such as reciever operating characteristic (ROC) curves for application in the longitudinal setting (Etzioni, Pepe, Longton, Hu and Goodman 1999; Slate and Turnbull 2000; Heagerty, Lumley and Pepe 2000). The goals of this manuscript are: to introduce new time-dependent sensitivity, specificity, and ROC concepts appropriate for survival regression models; to demonstrate the connection between time-dependent ROC methods and classical concordance summaries such as Kendall's tau or the "c index" (Harrell, Lee and Mark 1996); and to show how standard Cox regression estimation methods directly provide the ingredients needed to calculate the proposed time-dependent accuracy summaries.

\subsection{Notation}

Let $T_{i}$ be the survival time for subject $i$, and assume that we only observe the minimum of $T_{i}$ and $C_{i}$, where $C_{i}$ represents an independent censoring time. Define the follow-up time $X_{i}=$ $\min \left(T_{i}, C_{i}\right)$, and let $\Delta_{i}=1\left(T_{i} \leq C_{i}\right)$ denote the censoring indicator. The survival time $T_{i}$ can also be represented through the counting process, $N_{i}^{*}(t)=1\left(T_{i} \leq t\right)$, or the corresponding increment, $d N_{i}^{*}(t)=N_{i}^{*}(t)-N_{i}^{*}(t-)$. Note that we focus on the counting process $N_{i}^{*}(t)$ which is defined solely

in terms of the survival time $T_{i}$ rather than the more common notation $N_{i}(t)=1\left(X_{i} \leq t, \Delta_{i}=1\right)$ which depends on the censoring time (Fleming and Harrington 1991). Let $R_{i}(t)=1\left(X_{i} \geq t\right)$ denote 
the at-risk indicator. We also assume that for each subject we have a collection of time-invariant covariates, $\boldsymbol{Z}_{i}=\left(Z_{i 1}, Z_{i 2}, \ldots, Z_{i p}\right)$.

We focus here on using Cox model methods to both generate a model score, and to evaluate the prognostic potential of the model score. However, the evaluation methods that we propose can be used to summarize the accuracy of a prognostic score generated through any alternative regression or predictive method and in this case varying-coefficient methods (Hastie and Tibshirani 1993) such as locally weighted partial likelihood estimation (Cai and Sun 2003) provide a convenient approach for estimating key accuracy summaries. Therefore, we briefly introduce the relevant aspects of partial likelihood estimation. Under the proportional hazards assumption, $\lambda\left(t \mid \boldsymbol{Z}_{i}\right)=\lambda_{0}(t) \exp \left(\boldsymbol{Z}_{i}^{T} \boldsymbol{\beta}\right)$, where $\lambda\left(t \mid \boldsymbol{Z}_{i}\right)=\lim _{\delta \rightarrow 0} \delta^{-1} P\left[T_{i} \in[t, t+\delta) \mid \boldsymbol{Z}_{i}, T_{i} \geq t\right]$. The partial likelihood score equations can be writtten as:

$$
\mathbf{0}=\sum_{i} \Delta_{i}\left[\boldsymbol{Z}_{i}-\left(\sum_{k} \pi_{k}\left(\boldsymbol{\beta}, X_{i}\right) \boldsymbol{Z}_{k}\right)\right]
$$

where $\pi_{k}(\boldsymbol{\beta}, t)=R_{k}(t) \cdot \exp \left(\boldsymbol{Z}_{k}^{T} \boldsymbol{\beta}\right) / W(t)$, with $W(t)=\sum_{j} R_{j}(t) \cdot \exp \left(\boldsymbol{Z}_{j}^{T} \boldsymbol{\beta}\right)$. Solving these equations yields the consistent and asymptotically normal maximum partial likelihood estimator (MPLE) $\widehat{\boldsymbol{\beta}}$ (Cox 1972).

\subsection{Proportion of Variance Approaches}

Two main approaches exist for characterizing the proportion of variation explained by a survival model. Schemper and Henderson (2000) overview an approach where the survival time is characterized by a counting process representation, $N_{i}^{*}(t)=1\left(T_{i} \leq t\right)$, and time integrated variances are used to form the summary measure. Alternatively, O'Quigley and Xu (2001) consider the proportion of variation in the covariate, $Z_{i}$, that is explained by the survial time $T_{i}$.

Schemper and Henderson (2000) build on earlier work that extends $R^{2}$ to Cox regression. Their approach focuses on using the counting process, $N_{i}^{*}(t)$, and marginal and conditional expectations given by the survival functions $S(t)=E\left[1-N_{i}^{*}(t)\right]$ and $S\left(t \mid \boldsymbol{Z}_{i}\right)=E\left[1-N_{i}^{*}(t) \mid \boldsymbol{Z}_{i}\right]$ respectively. Since the vital status indicator $N_{i}^{*}(t)$ is a binary variable, Schemper and Henderson (2000) propose using the marginal variance $S(t)[1-S(t)]$ and the conditional variance $S\left(t \mid \boldsymbol{Z}_{i}\right)\left[1-S\left(t \mid \boldsymbol{Z}_{i}\right)\right]$ to characterize the proportion of variation explained by the covariates $\boldsymbol{Z}_{i}$. In particular, a finite time 
range $(0, \tau)$ is considered and time-average variances are formed

$$
\begin{aligned}
D(\tau) & =\int_{0}^{\tau} S(t)[1-S(t)] \cdot f(t) d t / \int_{0}^{\tau} f(t) d t \\
D_{\boldsymbol{Z}}(\tau) & =\int_{0}^{\tau} E_{\boldsymbol{Z}}\{S(t \mid \boldsymbol{Z})[1-S(t \mid \boldsymbol{Z})]\} \cdot f(t) d t / \int_{0}^{\tau} f(t) d t
\end{aligned}
$$

where $f(t)$ is the marginal density of $T_{i}$. Our representation above differs by a factor of 2 from the proposal of Schemper and Henderson (2000) as they also consider the mean absolute deviation, $E\left[\left|N_{i}^{*}(t)-S(t)\right|\right]=2 \cdot S(t)[1-S(t)]$. Finally, the summary $V(\tau)=\left[D(\tau)-D_{Z}(\tau)\right] / D(\tau)$ is proposed as the proportion of variation explained by covariates. Our approach similarly views survival data through the counting process representation, $N_{i}^{*}(t)$, but since $N_{i}^{*}(t)$ is a binary outcome we explore the extension of standard binary response accuracy summaries such as ROC curves rather than considering an extension of $R^{2}$.

O'Quigley and $\mathrm{Xu}$ (2001) also develop $R^{2}$ summaries for Cox regression. In their approach the role of survival time and covariate are reversed, and the proportion of variation in the covariate that is explained by survival is proposed. The authors exploit partial likelihood estimation methods since this provides model-based estimates of the distribution of covariates conditional on the survival time. Focusing on a scalar covariate, Xu and O'Quigley (2000) show that $\pi_{i}(\beta, t)=R_{i}(t) \exp \left(Z_{i} \beta\right) / W(t)$ can be used to estimate the distribution of the covariate, $Z_{i}$, con-

ditional on the event occuring at time $t, \widehat{P}\left(Z_{i} \leq z \mid T_{i}=t\right)=\sum_{j} \pi_{j}(\beta, t) \cdot \mathbf{1}\left(Z_{j} \leq z\right)$. O'Quigley and $\mathrm{Xu}(2001)$ obtain estimates of the conditional variance $\operatorname{var}\left(Z_{i} \mid T_{i}=t\right)$ and propose a global summary by integrating estimates of the marginal and conditional variance over the survival distribution. Our approach is similar to O'Quigley and Xu (2001) in that we also use $\pi_{i}(\boldsymbol{\beta}, t)$ but rather than computing variances we estimate time-dependent versions of sensitivity and specificity defined in the following section.

\subsection{Overview}

In section 2 we briefly review ROC methods proposed for summarizing the accuracy of a prognostic marker or model when the outcome of interest is a survival time. We then develop new definitions of time-dependent sensitivity and specificity that are strongly connected to partial likelihood concepts. 
Time-dependent accuracy measures can be used to calculate time-specific ROC curves, and timespecific area under the curve (AUC) summaries. We show that a global concordance measure is the integral, or weighted average, of time-specific AUC measures. In section 3 we discuss estimation of time-dependent ROC and AUC summaries and provide a method that is applicable to a proportional hazards model, and a more general method that can be used to characterize any scalar prognostic score even if proportional hazards does not obtain. Finally, in section 4 we analyze two well known data sets: the Mayo PBC data (Fleming and Harrington 1991); and the VA lung cancer data (Kalbfleisch and Prentice 2002). We conclude the manuscript with a brief discussion.

\section{Censored Survival and Predictive Accuracy}

When outcomes $Y_{i}$ are binary the accuracy of a prediction or classification rule is typically summarized through correct classification rates defined as sensitivity, $P\left(\widehat{p}_{i}>c \mid Y_{i}=1\right)$, and specificity, $P\left(\widehat{p}_{i} \leq c \mid Y_{i}=0\right)$, where $\widehat{p}_{i}$ is a prediction, and $c$ is a criterion for indicating a positive prediction. When no apriori value of $c$ is indicated the full spectrum of sensitivities and specificities can be characterized using an ROC curve that plots the "true positive rate" (sensitivity) versus the "false positive rate" (1-specifity) for all $c \in(-\infty,+\infty)$.

An ROC curve provides complete information on the set of all possible combinations of true positive and false positive rates, but is also more generally useful as a graphical characterization of the magnitude of separation between the case and control marker distributions. If case measurements and control measurements have no overlap then the ROC curve takes the value 1 (perfect true positive rate) for any false positive rate greater than 0 . In this situation the marker is perfect at discriminating between cases and controls. Alternatively, if the case and control distributions are identical then the ROC curve lies on the 45 degree line indicating that the marker is useless for separating cases from controls.

The area under the ROC curve, or AUC, is known to represent a measure of concordance between the marker and the disease status indicator (Hanley and McNeil 1982). Specifically, the AUC measures the probability that the marker value for a randomly selected case exceeds the marker value for a randomly selected control and is directly related to the Mann-Whitney U statistic (Hanley and McNeil 1982; Pepe 2003). Finally, ROC curves are particularly useful for comparing the 
discrimatory capacity of different potential biomarkers. For example, if for each value of specificity one marker always has a higher sensitivity, then this marker will be a uniformly better diagnostic measurement. See Zhou, McClish and Obuchowski (2002) or Pepe (2003) for more discussion of ROC analysis.

In this section we first review previous proposals for generalizing the concepts of sensitivity and specificity for application to survival endpoints. Definitions of sensitivity and specificity are given in terms of the actual survival time $T_{i}$. Censoring needs to be addressed for valid estimation. We then show that a certain choice of time-dependent true positive and false positive definitions lead to time-dependent ROC curves and time-dependent AUC summaries that are directly related to a previously proposed concordance summary for survival data.

\subsection{Extensions of Sensitivity and Specificity}

For survival data there are several potential extensions of cross-sectional sensitivity and specificity. Rather than a simple binary outcome, $Y_{i}=1$, a survival time can be viewed as a time-varying binary outcome by focusing on the counting process representation $N_{i}^{*}(t)=\mathbf{1}\left(T_{i} \leq t\right)$. Accuracy extensions are classified according to whether the "cases" used to define time-dependent sensitivity are incident cases where $T_{i}=t$, or equivalently $d N_{i}^{*}(t)=1$, is used to define cases for time $t$, or cumulative cases where $T_{i} \leq t$ or $N_{i}^{*}(t)=1$ is used. We also consider whether "controls" are static defined as subjects with $T_{i}>t^{\star}$ for a fixed value of $t^{\star}$, or whether controls are dynamic and defined for time $t$ as those subjects with $T_{i}>t$. We use the superscipts $\mathbb{C}$ and $\mathbb{I}$ to denote different definitions of sensitivity, and use the superscripts $\mathbb{D}$ and $\overline{\mathbb{D}}$ to denote different definitions of specificity. In this section we focus on a scalar marker value $M_{i}$ that is used as a predictor of death. When our interest is in the accuracy of a regression model we will use $M_{i}=\boldsymbol{Z}_{i}^{T} \boldsymbol{\beta}$.

Cumulative / Dynamic: For a baseline marker value, $M_{i}$, Heagerty et al. (2000) proposed versions of time-dependent sensitivity and specificity using the definitions:

$$
\begin{aligned}
& \text { sensitivity }^{\mathbb{C}}(c, t): P\left(M_{i}>c \mid T_{i} \leq t\right)=P\left(M_{i}>c \mid N_{i}^{*}(t)=1\right) \\
& \text { on of Blostatistics } \\
& \text { specificity } \mathbb{D}(c, t): P\left(M_{i} \leq c \mid T_{i}>t\right)=P\left(M_{i} \leq c \mid N_{i}^{*}(t)=0\right) .
\end{aligned}
$$


Using this approach, at any fixed time $t$ the entire population is classified as either a case or a control on the basis of vital status at time $t$. Also, each individual plays the role of a control for times $t<T_{i}$ but then contributes as a case for later times, $t \geq T_{i}$. Cumulative / Dynamic accuracy summaries are most appropriate when a specific time $t^{\prime}$ (or a small collection of times $\left.t_{1}^{\prime}, t_{2}^{\prime}, \ldots, t_{m}^{\prime}\right)$ is important and scientific interest lies in discriminating between subjects that die prior to a given time $t^{\prime}$ and those that survive beyond $t^{\prime}$. ROC curves are defined as $R O C_{t}^{\mathbb{C} / \mathbb{D}}(p)=$ $T P_{t}^{\mathbb{C}}\left\{\left[F P_{t}^{\mathbb{D}}\right]^{-1}(p)\right\}$ where $T P_{t}^{\mathbb{C}}(c)=P\left(M_{i}>c \mid N_{i}^{*}(t)=1\right), F P_{t}^{\mathbb{D}}(c)=P\left(M_{i}>c \mid N_{i}^{*}(t)=0\right)$, and $\left[F P_{t}^{\mathbb{D}}(p)\right]^{-1}=\inf _{c}\left\{c: F P_{t}^{\mathbb{D}}(c) \leq p\right\}$. In the absence of censoring $R O C_{t}^{\mathbb{C} / \mathbb{D}}(p)$ can be estimated using the empirical distribution of the marker separately among cases and controls. With censored survival times Heagerty et al. (2000) develop a non-parametric estimator based on the nearest neighbor bivariate distribution estimator of Akritas (1994). A substantive application that demonstrates use of Cumulative / Dynamic ROC curves for a Cox regression model can be found in Fan, Au, Heagerty, Deyo, McDonell and Fihn (2002).

Incident / Static: Etzioni et al. (1999), and Slate and Turnbull (2000) adopt an alternative definition of time-dependent sensitivity and specificity using:

$$
\begin{gathered}
\text { sensitivity }^{\mathbb{I}}(c, t): P\left(M_{i}>c \mid T_{i}=t\right)=P\left(M_{i}>c \mid d N_{i}^{*}(t)=1\right) \\
\operatorname{specificity}^{\overline{\mathbb{D}}}\left(c, t^{\star}\right) \quad: \quad P\left(M_{i} \leq c \mid T_{i}>t^{\star}\right)=P\left(M_{i} \leq c \mid N_{i}^{*}\left(t^{\star}\right)=0\right)
\end{gathered}
$$

where $d N_{i}^{*}(t)=N_{i}^{*}(t)-N_{i}^{*}(t-)$. Using this definition each subject does not change disease status and is treated as either a case or a control. Cases are stratified according to the time at which the event occurs (incident) and controls are defined as those subjects who are event-free through a fixed follow-up period, $\left(0, t^{\star}\right)$ (static). These definitions facilitate the use of standard regression approaches for characterizing sensitivity and specificity since the event time, $T_{i}$, can simply be used as a covariate. To estimate the quantiles of the conditional distribution of the marker, $M_{i}$, given the event time, $T_{i}=t$, Etzioni et al. (1999) and Slate and Turnbull (2000) consider parametric methods that assume a normal conditional distribution, but which allow the mean and variance to be functions of the measurement time, disease status, and the event time for the cases. Cai, Pepe, 
Lumley, Zheng and Jenny (2003) propose semi-parametric methods for estimating time-dependent sensitivity and specificity when the event time is censored. Recently Zheng and Heagerty (2003) have proposed use of regression quantile methods which relax the parametric distributional assumptions of previous Incident / Static methods.

Incident / Dynamic: In this manuscript we focus on the following definitions of sensitivity and specificity:

$$
\begin{aligned}
& \text { sensitivity }^{\mathbb{I}}(c, t): P\left(M_{i}>c \mid T_{i}=t\right)=P\left(M_{i}>c \mid d N_{i}^{*}(t)=1\right) \\
& \text { specificity }^{\mathbb{D}}(c, t): P\left(M_{i} \leq c \mid T_{i}>t\right)=P\left(M_{i} \leq c \mid N_{i}^{*}(t)=0\right) .
\end{aligned}
$$

Using this approach a subject can play the role of a control for an early time, $t<T_{i}$, but then play the role of case when $t=T_{i}$. This dynamic status parallels the multiple contributions that a subject can make to the partial likelihood function. Here sensitivity measures the expected fraction of subjects with marker greater than $c$ among the sub-population of individuals who die at time $t$, while specificity measures the fraction of subjects with a marker less than or equal to $c$ among those who survive beyond time $t$. Incident sensitivity and dynamic specificity are defined by dichotomizing the risk set at time $t$ into those observed to die (cases) and those observed to survive (controls). In section 3 we discuss how the observed marker data among risk sets can be used to estimate time-dependent accuracy concepts.

Incident sensitivity and dynamic specificity have some appealing characteristics relative to the alternative definitions. First, incident sensitivity and dynamic specificity are based on classification of the risk set at time $t$ into case(s) and controls and are therefore a natural companion to hazard models. Second, the definitions easily allow extension to time-dependent covariates using $P\left[M_{i}(t)>\right.$ $\left.c \mid T_{i}=t\right]$ to define incident sensitivity and $P\left[M_{i}(t) \leq c \mid T_{i}>t\right]$ to define dynamic specificity with a longitudinal marker $M_{i}(t)$. Use of cumulative sensitivity does not permit a time-varying marker. Finally, use of incident sensitivity and dynamic specificity allow both time-specific accuracy summaries and, as shown in section 2.3, allow time-averaged summaries which directly relate to a familiar global concordance measure. In contrast, methods have not been proposed for meaningfully 
averaging the time-specific Incident/Static or Cumulative/Dynamic accuracy summaries.

\subsection{Time-dependent ROC Curves}

After selecting definitions for time-dependent sensitivity and specificity, ROC curves can be computed and interpreted. In this manuscript we focus on Incident / Dynamic ROC curves defined as the function $R_{t} C_{t}^{\mathbb{I} / \mathbb{D}}(p)$ where $p$ denotes the false positive rate, $p=1-\operatorname{specificity}^{\mathbb{D}}\left(c^{p}, t\right)=P\left(M_{i}>\right.$ $\left.c^{p} \mid T_{i}>t\right)$, and $R O C_{t}^{\mathbb{I} / \mathbb{D}}(p)$ is the sensitivity that is obtained using the threshold $c^{p}$ defined above, or $\operatorname{ROC}_{t}^{\mathbb{I} / \mathbb{D}}(p)=$ sensitivity $^{\mathbb{I}}\left(c^{p}, t\right)=P\left(M_{i}>c^{p} \mid T_{i}=t\right)$. Using the true and false positive rate functions $T P_{t}^{\mathbb{I}}(c)=$ sensitivity $^{\mathbb{I}}(c, t)$ and $F P_{t}^{\mathbb{D}}(c)=1-$ specificity $^{\mathbb{D}}(c, t)$ allows the ROC curve to be written as the composition of $T P_{t}^{\mathbb{I}}(c)$ and the inverse function $\left[F P_{t}^{\mathbb{D}}\right]^{-1}(p)=c^{p}$ :

$$
R O C_{t}^{\mathbb{I} / \mathbb{D}}(p)=T P_{t}^{\mathbb{I}}\left\{\left[F P_{t}^{\mathbb{D}}\right]^{-1}(p)\right\}
$$

for $p \in[0,1]$. We use the notation $A U C(t)=\int_{0}^{1} R O C_{t}^{\mathbb{I} / \mathbb{D}}(p) d p$ to denote the area under the Incident / Dynamic ROC curve for time $t$.

\subsection{Time-dependent AUC and Concordance}

In the previous subsection we discussed how ROC methods can be used to characterize the ability of a marker to distinguish cases at time $t$ from controls at time $t$. However, in many applications no apriori time $t$ is identified, and a global accuracy summary is desired. In this subsection we show how time-dependent ROC curves are related to a standard "concordance" summary. The global summary we adopt is

$$
C=P\left[M_{j}>M_{k} \mid T_{j}<T_{k}\right]
$$

which indicates the probability that the subject who died at the earlier time has a larger value of the marker. This is not the usual form (ie. $P\left[M_{j}>M_{k} \mid T_{j}>T_{k}\right]$ ) but reflects the conventions for ROC analysis.

In order to understand the relationship between this discrimination summary and ROC curves we assume independence of observations $\left(M_{j}, T_{j}\right)$ and $\left(M_{k}, T_{k}\right)$, and assume that $T_{j}$ is continuous such that $P\left(T_{k}=T_{j}\right)=0$. We use $P(x)$ to denote probability or density depending on the context. These assumptions imply that the concordance summary $C$ is a weighted average of the area under 
time-specific ROC curves:

$$
\begin{aligned}
P\left[M_{j}>M_{k} \mid T_{j}<T_{k}\right]= & 2 \int_{t} P\left[\left\{M_{j}>M_{k}\right\} \mid\left\{T_{j}=t\right\} \cap\left\{t<T_{k}\right\}\right] \times P\left[\left\{T_{j}=t\right\} \cap\left\{t<T_{k}\right\}\right] d t \\
= & \int_{t} \operatorname{AUC}(t) \cdot w(t) d t=E_{T}[\operatorname{AUC}(T) \times 2 \times S(T)] \\
& \quad \text { with } w(t)=2 \cdot f(t) \cdot S(t) .
\end{aligned}
$$

In this notation $\mathrm{AUC}(t)$ is based on the Incident / Dynamic definition of sensitivity and specificity, $A U C(t)=P\left(M_{j}>M_{k} \mid T_{j}=t, T_{k}>t\right)$. See the Appendix for a derivation.

In practice we would typically restrict attention to a fixed follow-up period $(0, \tau)$. The concordance summary can be modified to account for finite follow-up:

$$
C^{\tau}=\int_{0}^{\tau} A U C(t) \cdot w^{\tau}(t) d t
$$

where $w^{\tau}(t)=2 \cdot f(t) \cdot S(t) / W^{\tau}, W^{\tau}=\int_{0}^{\tau} 2 \cdot f(t) \cdot S(t) d t=1-S^{2}(\tau)$. The restricted concordance summary remains a weighted average of the time-specific AUCs but with the weights rescaled such that they integrate to 1.0 over the range $(0, \tau)$. The interpretation of $C^{\tau}$ is a slight modification of the original concordance where:

$$
C^{\tau}=P\left[M_{j}>M_{k} \mid T_{j}<T_{k}, T_{j}<\tau\right]
$$

Thus $C^{\tau}$ is the probability that the predictions for a random pair of subjects are concordant with their outcomes, given that the smaller event time occurs in $(0, \tau)$.

The concordance summary $C$ is directly related to Kendall's tau. Specifically, $C=K / 2+1 / 2$ where $K$ denotes Kendall's tau (see Agresti (2002), p.60 for definition). Korn and Simon (1990) and Harrell et al. (1996) discuss use of Kendall's tau with survival data and propose modifications to account for censored observations.

\subsection{Example: Gaussian marker and log-normal disease time}

To illustrate time-dependent accuracy concepts we consider a simple example where the marker $M_{i}$ and the $\log$ of survival time $\log \left(T_{i}\right)$ follow a bivariate normal distribution. By convention we consider a higher marker value as indicative of earlier disease onset and therefore explore bivariate distributions with a negative correlation between the marker and $\log ($ time). 
If $\left[M_{i}, \log \left(T_{i}\right)\right]$ has a bivariate normal distribution with mean $(0,0)$ and unit standard deviations then time-dependent incident sensitivity and cumulative 1-specificity are:

$$
\begin{aligned}
P\left(M_{i}>c \mid d N_{i}^{*}(t)=1\right) & =T P_{t}^{\mathbb{I}}(c)=\Phi\left[\frac{\rho \cdot \log (t)-c}{\sqrt{\left(1-\rho^{2}\right)}}\right] \\
P\left(M_{i}>c \mid N_{i}^{*}(t)=0\right) & =F P_{t}^{\mathbb{D}}(c)=\frac{S_{2}^{\mathcal{N}}[c, \log (t) ; \rho]}{\Phi[-\log (t)]}
\end{aligned}
$$

where $\Phi(x)=P(X<x)$ for $X \sim \mathcal{N}(0,1)$ and $S_{2}^{\mathcal{N}}[x, y ; \rho]=P(X>x, Y>y)$ for $(X, Y)$ bivariate mean 0 (unit) normal with correlation $\rho$.

Figure 1 panel (a) shows Incident/Dynamic ROC curves for $\rho=-0.8$. The solid line corresponds to $t=\exp (-2)$ and has an AUC of 0.923 indicating very good separation between the distribution for $M_{i}$ among subjects with $T_{i}=\exp (-2)$ as compared to the marker distribution for subjects with $T_{i}>\exp (-2)$. Furthermore, if the threshold value $c^{10 \%}=1.19$ were used to indicate a positive test then by definition only $10 \%$ of the controls (ie. $\log \left(T_{i}\right)>-2$ ) would have a value of $M_{i}$ greater than 1.19. The ROC plot shows that for this false positive rate of $10 \%$ a sensitivity, or true positive rate, of $75 \%$ can be obtained: $T P_{t}^{\mathbb{I}}(1.19)=0.752$. If we consider a later time such as $\log (t)=0$ we find less overall discrimination with an AUC of 0.741. Again, specific operating points can be identified, for example, the ROC curve shows that if the false positive rate is again controlled at $10 \%$ then a true positive rate of only $30 \%$ is now obtained (here $c^{10 \%}=0.320$ ). One of the key advantages of an ROC curve is that it facilitates comparisons across different conditions in terms of the sensitivity of a marker where the specificity is controlled at a fixed level for each condition. In this example we have evaluated the variation in sensitivity over time while controlling 1-specificity at $10 \%$.

In Figure 1 panel (b) we show the $A U C(t)$ functions for different values of $\rho$. For each value of $\rho$ we find a decreasing $A U C(t)$ with increasing time. In addition, with decreasing correlation between the marker and the disease time we find uniformly decreasing values for $A U C(t)$. A global accuracy summary can be obtained using $C$ which integrates $A U C(t)$ using the weight function proportional to $2 \cdot f(t) \cdot S(t)$. Figure $1(\mathrm{~b})$ also displays the weight function which for this example is $w(t)=2 \cdot \phi(t)[1-\Phi(t)]$ where $\phi(x)$ and $\Phi(x)$ are the standard normal density and distribution functions respectively. In this bivariate normal situation there exists an analytical solution for the 
concordance: $C=\sin ^{-1}(-\rho) / \pi+0.5$. For $\rho=-0.9$ we find $C=0.827$ while with $\rho=-0.6$ we find $C=0.703$. Therefore, when the marker $M_{i}$ and log survival time have a correlation of -0.9 there is a $82.7 \%$ chance that for a random pair of observations the marker value for the earlier survival time is greater than the marker value for the larger survival time. This concordance probability is reduced to $70.3 \%$ when $\rho=-0.6$.

\section{Estimation of Incident / Dynamic (I/D) Time-dependent Accuracy}

In this section we propose methods for estimation of time-dependent accuracy summaries using a single scalar marker $M_{i}$. When interest is in the accuracy of a survival regression model we propose using the linear predictor as a scalar marker, $M_{i}=\boldsymbol{Z}_{i}^{T} \boldsymbol{\beta}$, and then using non-parametric or semi-parametric methods to characterize the time-dependent sensitivity and specificity of the model score. In particular we discuss how the Cox model and partial likelihood concepts can be conveniently used to provide semi-parametric estimates of Incident / Dynamic accuracy. However, the methods that we propose do not require the model score, $M_{i}$, to be derived from a proportional hazards model and are potentially applicable for any prognostic scale.

\subsection{Estimation: $T P_{t}^{\mathbb{I}}(c)$ and $F P_{t}^{\mathbb{D}}(c)$ Under Proportional Hazards}

Properties of the partial likelihood function make estimation of Incident / Dynamic ROC curves a natural companion to Cox regression. Here we assume that the censoring time $C_{i}$ is independent of the failure time $T_{i}$ and marker $M_{i}$. To clearly distinguish between the general model score, $M_{i}=\boldsymbol{Z}_{i}^{T} \boldsymbol{\beta}$, and a Cox model that uses this score we denote $\gamma$ as the proportional hazards regression parameter $\lambda\left(t \mid M_{i}\right)=\lambda_{0}(t) \exp \left(M_{i} \gamma\right)$. It is well known that under a proportional hazards model the weights, $\pi_{i}(\gamma, t)=R_{i}(t) \cdot \exp \left(M_{i} \gamma\right) / W(t)$ introduced in section 1.1, are used to compute an estimate of the expected value of the marker given failure:

$$
\widehat{E}\left(M_{i} \mid T_{i}=t\right)=\sum_{k} M_{k} \cdot \pi_{k}(\gamma, t)
$$

However, Xu and O'Quigley (2000) show that these weights can also be used to estimate the distribution of the covariate conditional on death at time $t$ :

$$
\widehat{T P}_{t}^{\mathbb{I}}(c)=\widehat{P}\left(M_{i}>c \mid T_{i}=t\right)=\sum_{k} 1\left(M_{k}>c\right) \cdot \pi_{k}(\gamma, t)
$$


where the estimate $\widehat{P}\left(M_{i}>c \mid T_{i}=t\right)$ is a consistent estimator when the Cox model for $M_{i}$ holds. Estimation of $\gamma$ using partial likelihood provides a semi-parametric estimate for $T P_{t}^{\mathbb{I}}(c)$. An empirical estimator can be used for $F P_{t}^{\mathbb{D}}(c)$ :

$$
\widehat{F P}_{t}^{\mathbb{D}}(c)=\widehat{P}\left(M_{i}>c \mid T_{i}>t\right)=\sum_{k} 1\left(M_{k}>c\right) \cdot R_{k}(t+) / W^{R}(t+)
$$

where $R_{k}(t+)=\lim _{\delta \rightarrow 0} R_{k}(t+|\delta|)$, and $W^{R}(t+)=\sum_{k} R_{k}(t+)$. The term $W^{R}(t+)$ denotes the size of the "control set" at time $t$ where we define the control set as the risk set minus subjects who fail at time $t$. Essentially, $\widehat{F P}_{t}^{\mathbb{D}}(c)$ is the empirical distribution function for marker values among the control set, and $\widehat{T P}_{t}^{\mathbb{I}}(c)$ is an exponential tilt of the empirical distribution function for the marker among risk set subjects (Anderson 1979).

\subsection{Estimation: $T P_{t}^{\mathbb{I}}(c)$ and $F P_{t}^{\mathbb{D}}(c)$ Under Non-proportional Hazards}

In order to use equation (3.1) to estimate incident sensitivity the proportional hazards assumption must be satisfied. However, this aspect can be relaxed by adopting a varying-coefficient model of the form $\lambda\left(t \mid M_{i}\right)=\lambda_{0}(t) \exp \left[M_{i} \gamma(t)\right]$. The time-varying coefficient function $\gamma(t)$ can be estimated either in a 1-step fashion based on routine Cox model residuals, or through locally weighted partial likelihood methods. Note if proportional hazards does obtain then $\gamma(t) \equiv 1$ when $M_{i}=\boldsymbol{Z}_{i}^{T} \boldsymbol{\beta}$.

Grambsch and Therneau (1994) describe residual-based methods for assessing the proportional hazards model that can also be used to obtain estimates of time-varying coefficient functions. In order to define the residuals we adopt the following notation: $S^{(p)}(\boldsymbol{\beta}, t)=\sum_{k} R_{k}(t) \exp \left(\boldsymbol{Z}_{k}^{T} \boldsymbol{\beta}\right) \cdot \boldsymbol{Z}_{k}^{\otimes p}$ where $\boldsymbol{Z}_{k}^{\otimes p}$ refers to $1, \boldsymbol{Z}_{k}$, and $\boldsymbol{Z}_{k} \boldsymbol{Z}_{k}^{T}$ for $p=0,1,2$ respectively. The "scaled Schoenfeld residuals" are defined for each observed ordered failure time, $t_{(j)}$, as the vector:

$$
r_{j}^{*}(\boldsymbol{\beta})=V^{-1}\left[\boldsymbol{\beta}, t_{(j)}\right]\left\{\boldsymbol{Z}_{(j)}-e\left[\boldsymbol{\beta}, t_{(j)}\right]\right\}
$$

where $e\left[\boldsymbol{\beta}, t_{(j)}\right]=S^{(1)}\left[\boldsymbol{\beta}, t_{(j)}\right] / S^{(0)}\left[\boldsymbol{\beta}, t_{(j)}\right], V\left[\boldsymbol{\beta}, t_{(j)}\right]=S^{(2)}\left[\boldsymbol{\beta}, t_{(j)}\right] / S^{(0)}\left[\boldsymbol{\beta}, t_{(j)}\right]-e\left[\boldsymbol{\beta}, t_{(j)}\right] e\left[\boldsymbol{\beta}, t_{(j)}\right]^{T}$, and $\boldsymbol{Z}_{(j)}$ denotes the covariate for the subject observed to die at time $t_{(j)}$. Grambsch and Therneau (1994) show that: (i) $E\left\{r_{j}^{*} \mid \mathcal{F}\left[t_{(j)}\right]\right\} \approx\left[\boldsymbol{\beta}(t)-\boldsymbol{\beta}_{0}\right]$; and (ii) $\operatorname{var}\left\{r_{j}^{*} \mid \mathcal{F}\left[t_{(j)}\right\} \approx V^{-1}\left[\boldsymbol{\beta}, t_{(j)}\right]\right.$, where $\boldsymbol{\beta}_{0}$ is the time-averaged coefficient and $\mathcal{F}(t)$ is the right-continuous filtration specifying the survival process history. These properties are used to obtain focused tests of proportionality, and to obtain 
estimates of the time-varying coefficient function, $\beta_{k}(t)$ corresponding to covariate $Z_{i, k}$. As a graphical diagnostic tool standard regression smoothing techniques are now commonly applied to the points $\left[t_{(j)}, \widehat{\beta}_{k}+r_{j, k}^{*}(\widehat{\boldsymbol{\beta}})\right]$ following a Cox model fit in order to obtain estimates of timedependent coefficient functions, $\beta_{k}(t)$.

For the evaluation of the accuracy of a marker, $M_{i}$, the smoothing of Schoenfeld residuals can be used to obtain a simple estimate of Incident/Dynamic $A U C(t)$ by exploiting standard Cox model output. First a Cox model of the form $\lambda_{0}(t) \exp \left(M_{i} \gamma\right)$ is fit, followed by use of regression smoothing methods to obtain $\widehat{\gamma}(t)$. Second, equation (3.2) can still be used to obtain estimates of false positive rates, and (3.1) can be now be evaluated using $\gamma(t)$ rather than a constant value $\gamma$ :

$$
\widehat{T P}_{t}^{\mathbb{I}}(c)=\widehat{P}\left(M_{i}>c \mid T_{i}=t\right)=\sum_{k} 1\left(M_{k}>c\right) \cdot \pi_{k}[\widehat{\gamma}(t), t]
$$

By using equation (3.3) we are adopting the flexible semi-parametric hazard model, $\lambda_{0}(t) \exp \left[M_{i} \gamma(t)\right]$, which no longer assumes proportionality but rather only assumes smoothly varying hazard ratios over time.

More formal flexible semi-parametric statistical methods can be used to estimate a varyingcoefficient hazard model and subsequently produce time-dependent accuracy summaries based on minimal model assumptions. For example, Hastie and Tibshirani (1993) discuss both smooth parametric methods and non-parametric penalized likelihood methods for estimating the function $\gamma(t)$ in the model $\lambda_{i}(t)=\lambda_{0}(t) \exp \left[M_{i} \gamma(t)\right]$. More recently Cai and Sun (2003) characterize the properties of locally weighted partial likelihood methods used to obtain varying coefficient estimates. Using kernel weights that are specified as a function of time, $t$, allows use of local-linear estimation methods. Cai and Sun (2003) prove the pointwise consistency and asymptotic normality of the resulting function estimator, $\widehat{\gamma}(t)$. Smooth parametric and/or non-parametric methods allow valid estimation of accuracy summaries such as $A U C(t)$ based on the minimal model assumptions since models of the form $\lambda_{i}(t)=\lambda_{0}(t) \exp \left[M_{i} \gamma(t)\right]$ only assume linearity in $M_{i}$ and smoothly varying hazard ratios over time. The linearity assumption can be relaxed by using a model with single or multiple transformations of $M_{i}$ and a vector of time-varying coefficients. 


\subsection{Estimation: $R O C_{t}^{\mathbb{I} / \mathbb{D}}(p), A U C(t)$, and $C^{\tau}$}

Given estimates of $T P_{t}^{\mathbb{I}}(c)$ and $F P_{t}^{\mathbb{D}}(c)$ the area under the ROC curve at time $t, A U C(t)$, and the integrated area, $C^{\tau}$, can be calculated. The estimated ROC curve is given as

$$
\widehat{R O C}_{t}^{\mathbb{I}} / \mathbb{D}(p)=\widehat{T P}_{t}^{\mathbb{I}}\left\{\left[\widehat{F P}_{t}^{\mathbb{D}}\right]^{-1}(p)\right\}
$$

where $\left[\widehat{F P}_{t}^{\mathbb{D}}\right]^{-1}(p)=\inf _{c}\left\{c: \widehat{F P}_{t}(c) \leq p\right\}$. The estimated $A U C(t)$ is simply $\widehat{A U C}(t)=\int \widehat{R O C}_{t}^{\mathbb{I} / \mathbb{D}}(p) d p$ estimated using standard numerical integration methods such as the trapezoid rule. Finally, the estimated concordance is given by

$$
\widehat{C}^{\tau}=\int \widehat{A U C}(t) \cdot \widehat{w}^{\tau}(t) d t
$$

where $\widehat{A U C}(t)$ is given above and $\widehat{w}^{\tau}(t)=2 \cdot \widehat{f}(t) \cdot \widehat{S}(t) /\left[1-\widehat{S}^{2}(\tau)\right]$. The Kaplan-Meier estimator can be used for $\widehat{S}(t)$, and a discrete approximation to $\widehat{f}(t)$ can be used based on the increments in the Kaplan-Meier estimator. If Kaplan-Meier is used to estimate $f(t)$ and $S(t)$ then $\widehat{A U C}(t)$ only needs to be evaluated at the observed failure times in order to calculate $\widehat{C}^{\tau}$.

\subsection{Inference for Incident / Dynamic Accuracy Summaries}

$\mathrm{Xu}$ and O'Quigley (2000) show that the estimator $\widehat{T P}_{t}^{\mathbb{I}}(c)$ given in equation (3.1) is consistent provided that the proportional hazards model obtains, and provided the independent observations are subject to independent censoring. Parallel arguments apply for the estimator obtained using a varying-coefficient model given in equation (3.3) whenever a consistent estimator of $\gamma(t)$ is used. Cai and Sun (2003) show that the locally-weighted MPLE is consistent under standard regularity conditions. In addition, since $\widehat{F P}_{t}^{\mathbb{D}}(c)$ is an empirical distribution function calculated over the control set (i.e. the risk set minus the case), consistency obtains provided the control set represents an unbiased sample (ie. independent censoring). Therefore, consistent estimates of time-dependent sensitivity and specificity and corresponding $A U C(t)$ and $C^{\tau}$ summaries are obtained under the proportional hazards assumption using equations (3.1) and (3.2), and under more general nonproportional hazards assumptions using equation (3.3). Finally, since the accuracy summaries are defined over the joint distribution of the marker $M_{i}$ and the survival time $T_{i}$, the non-parametric 
bootstrap of Efron (1979) based on resampling of observations $\left(M_{i}, X_{i}, \Delta_{i}\right)$ can be used to compute standard errors or to provide confidence intervals.

\subsection{Discrete Times and General Hazard Models}

Our motivation for developing tools to summarize predictive accuracy stems from interest in characterizing the prognostic potential of Cox models for continuous survival times. However, the basic time-dependent accuracy concepts and the estimation method outlined in section 3.2 generalizes to discrete survival times and/or alternative hazard regression models.

The key to estimation of $T P_{t}^{\mathbb{I}}(c)$ presented in sections 3.1 and 3.2 is that a hazard model can be used to reweight the empirical distribution of $M_{i}$ calculated over the risk set at time $t$. Equations (3.1) and (3.3) show specific details for Cox models. More generally, let $P\left(T_{i}=t \mid T_{i} \geq t, M_{i}\right)$ denote the hazard, where $P(t)$ represents either density for continuous survival times or probability for discrete times. A hazard regression model can be formulated as: $g\left[P\left(T_{i}=t \mid T_{i} \geq t, M_{i}\right)\right]=$ $\alpha(t)+M_{i} \beta(t)$ where $g(x)$ is a link function. The Cox model is a special case where: a log link is used; $\alpha(t)=\log \lambda_{0}(t)$; and $\beta(t) \equiv \beta$ under the proportional hazards assumption. Following arguments given in Xu and O'Quigley (2000) the general model implies:

$$
P\left(M_{i}=m \mid T_{i}=t\right) \quad \propto \quad g^{-1}[\alpha(t)+m \cdot \beta(t)] \times P\left(M_{i}=m \mid T_{i} \geq t\right)
$$

where $P(m)$ denotes either the marker density or probability depending on whether a continuous or discrete marker distribution is assumed. See the Appendix for a derivation. Equation (3.4) shows that $P\left(M_{i}=m \mid T_{i}=t\right)$ can be estimated from separate estimates of the hazard model and the distribution of the marker conditional on $T_{i} \geq t$. Therefore, the general estimation approach outlined in section 3.2 can be adopted for either discrete survival times or for general hazard regression models provided that consistent estimates of $[\alpha(t), \beta(t)]$ and $P\left(M_{i}=m \mid T_{i} \geq t\right)$ are available. Tied survival times impact choice of a method for estimating the hazard model parameters. In addition, with discrete survival times calculation of the concordace summary $C=$ $\int A U C(t) \cdot w(t) d t$ requires modification to account for the fact that $P\left(T_{j}=T_{k}\right) \neq 0$ and therefore the constant 2 in the weight $w(t)=2 \cdot f(t) \cdot S(t)$ needs to be computed as $1 / P\left(T_{j}<T_{k}\right)$. Finally, Cox models are convenient since the baseline hazard, $\alpha(t)=\log \lambda_{0}(t)$, drops out of (3.4) and is 
thus not required for estimation of $T P_{t}^{\mathbb{I}}(c)$.

\subsection{Simulations to Evaluate Incident / Dynamic Estimation}

In order to demonstrate the feasibility of using Cox regression methods and the marker distribution among risk sets for estimating Incident / Dynamic ROC curves and global concordance we conducted a set of simulation studies.

For each of $m=500$ simulated data sets a sample of $n=200$ marker values, $M_{i}$, and survival times, $T_{i}$, were generated such that $\left(M_{i}, \log T_{i}\right)$ is bivariate normal with a correlation of $\rho=-0.7$. An independent log-normal censoring time was generated to yield a fixed expected fraction of censored observations (either $20 \%$ or $40 \%$ censored). For each simulated data set we estimated the Incident / Dynamic $A U C(t)$ function and the concordance summary $C^{\tau}$ using the largest observed survival time to truncate follow-up time. For each simulated data set we applied four methods of estimation to the censored data: maximum likelihood assuming a bivariate normal distribution for the survival time and the marker; maximum partial likelihood using the Cox model which for this example incorrectly assumes proportional hazards; locally-weighted maximum partial likelihood (MPL) estimation for the model $\lambda_{0}(t) \exp \left[M_{i} \gamma(t)\right]$ using the method of Cai and Sun (2003); and simple local linear smoothing of the scaled Schoenfeld residuals. For both local MPL estimation and local linear smoothing we used an Epanechnikov kernel with a span of $n^{-1 / 5}$ where $n$ is the number of observations (Cai and Sun 2003).

In order to estimate $A U C(t)$ and $C^{\tau}$ using semi-parametric methods the model for the survival time conditional on the marker, $\lambda_{0}(t) \exp \left[M_{i} \gamma(t)\right]$, is combined with the observed marker distribution within each risk-set according to the methods described in section 3.2. We have adopted a survival model that assumes that the log hazard increases linearly in $M_{i}$ for each time $t$. The true data-generating model is actually non-linear with a concave risk function. Therefore for this simulation study our estimation used a first-order approximation to the true conditional hazard surface.

Table 1 displays the mean and standard deviation for the estimate of $A U C(t)$ at various values of $t$ when data are generated with $20 \%$ and with $40 \%$ censoring. When $20 \%$ of the observations 
are censored we find that the MLE for $A U C(t)$ has minimal bias for $\log (t)$ between -2 and 2 . Estimates based on the locally-weighted MPLE and the residual smoother yield approximately unbiased estimates for all but the most extreme values of time with some negative bias observed for both semi-parametric estimators. For example, at $\log (t)=-2$ the mean $\widehat{A U C}(t)$ using the locallyweighted MPLE is 0.860 (relative bias of $1-0.860 / 0.884=-3 \%$ ) and using the residual smoother the average is 0.881 (relative bias of $1-0.881 / 0.884<-1 \%$ ) while at $\log (t)=2$ the locally weighted MPLE mean estimate is 0.579 (relative bias $=1-0.579 / 0.598=-3 \%$ ) and for the residual smoother the mean is 0.573 (relative bias $=1-0.573 / 0.598=-4 \%$ ). As expected for local regression methods Table 1 shows that the non-parametric methods yield substantially greater variances for specific values of $t$ compared to the MLE.

Incorrectly assuming proportional hazards leads to biased estimates. Table 1 shows that the estimated $A U C(t)$ obtained using equation (3.1) with an estimated Cox model coefficient is negatively biased for $\log (t)<0$. For example, at $\log (t)=-2$ we obtain a negative bias of $1-0.743 / 0.884=$ $-16 \%$. For $\log (t)>0$ the estimates obtained using the Cox model and equation (3.1) are positively biased indicating that direct use of the proportional hazards assumption produces an estimated $A U C(t)$ curve that is flatter than the target with early underestimation and late overestimation.

When censoring is increased to $40 \%$ similar patterns are found for all estimators. Table 1 shows that the bias in $\widehat{A U C}(t)$ is slightly larger with increased censoring. For example at $\log (t)=2$ the mean estimate for the locally-weighted MPLE is 0.555 (relative bias of $1-0.555 / 0.598=-7 \%$ ) and for the residual smoother is 0.546 (relative bias of $1-0.546 / 0.598=-9 \%$ ). Therefore, even with $40 \%$ censoring the smooth semi-parametric methods appear to perform adequately.

Finally, Table 1 also shows the results for estimation of the global concordance summary $C^{\tau}$. In the simulations we estimate $C$ using the analytical results for the MLE: $\widehat{C}=\sin ^{-1}(-\widehat{\rho}) / 2+1 / 2$. For the methods that adopt a varying coefficient hazard model we set $\tau$ equal to the largest uncensored survival time in the observed data and therefore truncate follow-up at slightly different times for each simulated data set. However, even with $40 \%$ censoring the largest uncensored time had a median value of $\exp (2.30)$ with interquartile range of $\exp (2.04)$ to $\exp (2.65)$, and thus typically very little mass in the survival distribution is lost since $S[\exp (2.30)]=1-\Phi(2.30)=$ 
0.01. With $20 \%$ censoring the mean estimate for the MLE, locally-weighted MPLE, and residual smoother are 0.741 (s.d. $=0.016), 0.737$ (s.d. $=0.018)$, and 0.740 (s.d. $=0.018)$ respectively. In contrast the estimate obtained naively assuming proportional hazards is negatively biased with an average estimate of 0.720 (relative bias $=1-0.720 / 0.741=-3 \%$ ). These results suggest that the smooth semi-parametric methods yield little bias, and for this example exhibit high efficiency relative to the MLE. A similar pattern is seen with $40 \%$ censoring where slightly increased standard deviations are observed relative to results obtained with $20 \%$ censoring.

\section{Examples}

In this section we illustrate the Incident/Dynamic time-dependent ROC curves, the $A U C(t)$ plot, and the concordance summary $C^{\tau}$ using two well studied data sets.

\subsection{VA Lung Cancer Data}

Kalbfleisch and Prentice (2002) present and analyze Veteran's Administration (VA) lung cancer data from a clinical trial in which males with inoperable cancer were randomized to a standard treatment or a test therapy. Baseline covariates that were considered important predictors of mortality include: patient age; histological type of tumor; and a performance status measure known as the Karnofsky score. Schemper and Henderson (2000) use these covariates plus a treatment indicator and report an $R^{2}$ of $\widehat{V}=0.24$. This would suggest that the covariates explain only $24 \%$ of the time integrated variance in survival status.

For comparison we use the same covariates and Cox regression to create estimates of $R O C_{t}^{\mathbb{I} / \mathbb{D}}(p)$ for select $t$, the $A U C(t)$ function, and the concordance summary $C^{\tau}$. For our analysis we terminate follow-up at 500 days. Estimated model coefficients and standard errors are given in Table 2. Using the proportional hazards assumption we can employ equations (3.1) and (3.2) to estimate

time-specific I/D ROC curves, and then integrate the ROC curve to obtain $\widehat{A U C}(t)$. Estimates of $A U C(t)$ and pointwise $90 \%$ confidence intervals are displayed in Figure 2(a). Over the first 60 days of follow-up the $A U C(t)$ ranges between 0.66 and 0.73 . The substantive interpretation is: on any day, $t$, between 0 and 60 , the probability that a subject who dies on day $t$ having a model score greater than a subject who survives beyond day $t$ is at least 0.66 . The accuracy summaries suggest 
good short-term discriminatory potential of the model score. The estimated $A U C(t)$ function tends to decline over time to approximately 0.65 for $100<t<300$. Estimates of $A U C(t)$ also become increasingly variable over time due to the diminishing size of the risk set. Using a follow-up of $\tau=365$ days yields a concordance estimate of $\int_{0}^{\tau} \widehat{A U C}(t) \cdot \widehat{w}^{\tau}(t) d t=0.713$ with a standard error of 0.026 . This implies that conditional on one event occuring within the first year, the probability that the model score is larger for the subject with the smaller event time is $71.3 \%$. The concordance estimate $\widehat{C}^{\tau}$ is relatively modest in magnitude, but is significantly different from the null value of $0.50\left(95 \%\right.$ CI for $\left.C^{\tau}: 0.661,0.765\right)$.

To characterize the model score, $M_{i}=\boldsymbol{Z}_{i}^{T} \widehat{\boldsymbol{\beta}}$, using fewer assumptions we relax the proportional hazards assumption for $M_{i}$ by using a varying coefficient model: $\lambda_{0}(t) \exp \left[M_{i} \gamma(t)\right]$. Note that we are still focusing on use of the Cox model with a proportional hazards assumption to generate the model score, but are relaxing assumptions needed to characterize the model accuracy. This highlights the fact that different methods can be used for generating and evaluating a survival regression model score (linear predictor). For the VA lung cancer data we simply use a kernel smooth of the scaled Schoenfeld residuals to estimate $\gamma(t)$. The estimate of $\gamma(t)$ suggests a decreasing log relative hazard with increasing time (not shown).

Figure 2(b) shows estimates of $A U C(t)$ based on equations (3.2) and (3.3) which relax the proportional hazards assumption. First notice that the short-term accuracy of the model score remains good with $\widehat{A U C}(t)$ between 0.70 and 0.78 over the first 60 days of follow-up. Second, the discriminatory ability of the model score declines substantially over time, and estimates of $A U C(t)$ approach 0.50 after approximately 300 days, suggesting that the model score is essentially useless at discriminating incident cases from controls after 300 days. The one year concordance is estimated as $\widehat{C}^{\tau}=0.738$, a slight increase from the estimate obtained assuming proportional hazards. In this example the $A U C(t)$ curve is particularly useful for displaying the fact that the baseline model score is good at discriminating early cases from early controls, but is of decreasing prognostic utility with increasing temporal distance from the baseline measurement. Declining prognostic value is not surprising particularly since Karnofsky score is actually a time-varying health status measure, but only the baseline value is available for the regression model. Figure 3 shows select estimates 
of Incident/Dynamic ROC curves based on the varying-coefficient model. Similar to the plot of $A U C(t)$ the ROC curves show that predictive accuracy is uniformly decreasing with increasing time since baseline. For example, controlling the dynamic false positive rate at $20 \%$ leads to an incident sensitivity of $56 \%$ at 30 days, decreasing to $45 \%, 42 \%$, and $38 \%$ for 60,90 , and 120 days. The ROC curves also show detail regarding the trade-off between sensitivity and specificity. If a stricter false positive rate of $10 \%$ was desired then the corresponding sensitivity would only be $40 \%$ at 30 days and less than $30 \%$ for follow-up times of 60 days or greater.

\subsection{Mayo PBC Data}

Next we consider data from a randomized placebo controlled trial of the drug D-penicillamine (DPCA) for treatment of primary biliary cirrhosis (PBC) conducted at the Mayo Clinic between 1974 and 1984 (Fleming and Harrington 1991). Among the 312 subjects randomized to the study, 125 died by the end of the follow-up. Although the study established that DPCA is not effective for treatment of $\mathrm{PBC}$, the data have been used to develop a commonly used clinical prediction model. We use this example to illustrate how ROC curves and/or $A U C(t)$ summaries can be used to compare different model scores.

We first consider a Cox model containing 5 covariates: $\log$ (bilirubin), albumin, $\log$ (prothrombin time), edema and age. Table 3 gives the regression estimates using the proportional hazard model with mortality as the response. Except for $\log ($ prothrombin time), all covariates are strong predictors of survival. The model has been used to create a widely used prognostic score. We now address the basic question: how well does the model score discriminate subjects that are likely to die from subjects that are likely to survive? In addition we consider whether the accuracy of the score changes over time. Using the fitted linear predictor from the Cox model, we construct Incident/Dynamic time-dependent ROC curves and associated summaries in order to characterize the accuracy of the "Mayo model". Figure 4(a) plots $A U C(t)$ evaluated at each failure time. The model score has very good discriminatory capacity for distinguishing those patients who die at time $t$ from those who live beyond time $t$. The accuracy is especially good for follow-up times less than 1000 days, with early $A U C(t)$ estimates exceeding 0.85 . The accuracy of the model score gradually 
decreases with time. Based on $\widehat{A U C}(t)$ and the Kaplan-Meier estimator of the marginal survival distribution we estimate a concordance summary, $C^{\tau}$, of 0.80 , with $\tau$ fixed at 4000 days for this and subsequent analysis.

To quantify the impact of a single covariate on the accuracy of prediction we fit a second Cox regression model that does not include the covariate $\log ($ bilirubin). Table 3 displays coefficient estimates for this new four covariate model. The estimate of $C^{\tau}$ drops from 0.80 to 0.73 when $\log$ (bilirubin) is excluded from the model. In addition, we can use the estimated $A U C(t)$ curves shown in Figure 4(a) to quantify for each follow-up time $t$ the additional predictive accuracy that is obtained by using bilirubin in addition to the other model covariates. Relative to the 5 covariate model The estimated $A U C(t)$ for the 4 covariate model is approximately 0.10 units below the 5 covariate model $\widehat{A U C}(t)$ for $t$ between 0 and 2000 days.

We then relax the proportional hazard assumption and use the time-varying coefficient models as described in section 3.2 to characterize the accuracy of the model score $M_{i}=\boldsymbol{Z}_{i}^{T} \widehat{\boldsymbol{\beta}}$. The bottom panel of Figure 4 displays the AUC function based on the estimated time-varying coefficient obtained using locally-weighted MPL. Early estimates of $A U C(t)$ now exceed 0.90 and decline sharply to approximately 0.75 at 2000 days for the five covariate model and to less than 0.65 at 2000 days for the four covariate model. Using the estimated $A U C(t)$ reveals that the "Mayo model" is excellent at short term prediction but that the predictive accuracy declines to $\widehat{A U C}(t)<0.80$ by one year for the model without bilirubin, and to $\widehat{A U C}(t)<0.80$ by five years for the five covariate model. Finally, using the time-varying coefficient produces a global concordance summary of 0.80 for the five-covariate model and 0.72 for the model that excludes bilirubin.

\section{Discussion}

This manuscript introduces a new version of time-dependent sensitivity, specificity, and associated ROC curves that are useful for characterizing the predictive accuracy of a scalar marker, such as a derived model score, when the outcome is a censored survival time. We show that the area under the time-specific ROC curves can be plotted as a function of time to characterize temporal changes in accuracy, and can be integrated using the marginal distribution of the failure time to provide a global concordance summary. Incident sensitivity and dynamic specificity are shown to be easily estimated 
using a fitted hazard model and the empirical distribution of the marker data within risk sets. Using only routine Cox model output allows estimates of accuracy that assume proportional hazards and simple regression smoothing of scaled Schoenfeld residuals provides accuracy summaries appropriate for markers that do not satisfy proportional hazards. Simulations suggest that residual smoothing and locally-weighted partial likelihood estimators both provide feasible, and accurate estimates.

Time-dependent ROC curves offer an alternative to the use of $R^{2}$ extensions for survival data. However, the goal of an ROC analysis is to characterize the prognostic potential of a marker (or model) by focusing on correct classification rates. Methods that summarize the proportion of variation explained by covariates require a different estimation approach and have a different ultimate goal. Our methods also explicitly decouple the generation of a predictive score from the evaluation of prognostic accuracy. An investigator may use Cox regression to create a model score $M_{i}=\boldsymbol{Z}_{i}^{T} \boldsymbol{\beta}$ that is a time-invariant linear combination of baseline covariates $\boldsymbol{Z}_{i}$. However, using the flexible methods proposed in section 3.2 to evaluate the prognostic potential of $M_{i}$ does not require commitment to the proportional hazards assumption. A practical advantage of using $M_{i}=\boldsymbol{Z}_{i}^{T} \boldsymbol{\beta}$ is that a single "scoring" of the baseline covariates is conducted to generate $M_{i}$, but if proportional hazards is clearly violated then a more general model such as $\lambda_{0}(t) \exp \left[\boldsymbol{Z}_{i}^{T} \boldsymbol{\beta}(t)\right]$ may be appropriate, and would lead to a time-varying score $M_{i}(t)=\boldsymbol{Z}_{i}^{T} \boldsymbol{\beta}(t)$.

A number of aspects warrant additional research. First, estimation methods proposed in sections 3.1 and 3.2 assume that the censoring time is independent of the survival time. Relaxation to allow conditional independence given the marker, $M_{i}$, or covariates, $\boldsymbol{Z}_{i}$, would be useful. Second, we have proposed estimators that assume a prospective study design. Extension to case-cohort data may be important for characterizing the accuracy of markers for rare diseases. Third, development of analytical approximations that characterize the large sample distribution of the proposed estimators would facilitate approximate inference for time-dependent ROC curves, the $A U C(t)$ curve, or the concordance summary $C^{\tau}$. Finally, exploration of time-depenent accuracy methods with a longitudinal marker, $M_{i}(t)$, would be important for the common prospective medical setting in which predictive covariate information is updated over time. 


\section{REFERENCES}

Agresti, A. (2002), Categorical Data Analysis, Second Edition John Wiley \& Sons.

Akritas, M. G. (1994), "Nearest neighbor estimation of a bivariate distribution under random censoring," The Annals of Statistics, 22, 1299-1327.

Anderson, J. A. (1979), "Multivariate logistic compounds," Biometrika, 66, 17-26.

Cai, T., Pepe, M. S., Lumley, T., Zheng, Y., and Jenny, N. S. (2003), "The sensitivity and specificity of markers for event times," University of Washington Technical Report, (188), 1-30.

Cai, Z., and Sun, Y. (2003), "Local linear estimation for time-dependent coefficients in Cox's regression models," Scandinavian Journal of Statistics, 30, 93-111.

Cox, D. R. (1972), "Regression models and life-tables (with discussion)," Journal of the Royal Statistical Society, Series B, Methodological, 34, 187-220.

Efron, B. (1979), "Bootstrap methods: Another look at the jackknife," The Annals of Statistics, 7, 1-26.

Etzioni, R., Pepe, M., Longton, G., Hu, C., and Goodman, G. (1999), "Incorporating the time dimension in receiver operating characteristic curves: A case study of prostate cancer," Medical Decision Making, 19, 242-251.

Fan, V., Au, D., Heagerty, P., Deyo, R., McDonell, M., and Fihn, S. (2002), "Validation of case-mix measures derived from self-reports of diagnoses and health," Journal of Clinical Epidemiology, 55, 371-380.

Fleming, T. R., and Harrington, D. P. (1991), Counting processes and survival analysis John Wiley $\&$ Sons.

Grambsch, P. M., and Therneau, T. M. (1994), "Proportional hazards tests and diagnostics based on weighted residuals (Corr: 95V82 p668)," Biometrika, 81, 515-526.

Hanley, J. A., and McNeil, B. (1982), "The meaning and use of the area under the receiver operating characteristic (ROC) curve," Radiology, 143, 29-36.

Harrell, F. E., Lee, K. L., and Mark, D. B. (1996), "Multivariable prognostic models: Issues in developing models, evaluating assumptions and adequacy, and measuring and reducing errors," Statistics in Medicine, 15, 361-387.

Hastie, T., and Tibshirani, R. (1993), "Varying-coefficient models," Journal of the Royal Statistical Society, Series B, 55, 757-796. 
Heagerty, P. J., Lumley, T., and Pepe, M. S. (2000), "Time-dependent ROC curves for censored survival data and a diagnostic marker," Biometrics, 56(2), 337-344.

Kalbfleisch, J. D., and Prentice, R. L. (2002), The statistical analysis of failure time data John Wiley \& Sons.

Korn, E. L., and Simon, R. (1990), "Measures of explained variation for survival data," Statistics in Medicine, 9, 487-503.

O'Quigley, J., and Xu, R. (2001), Explained variation in proportional hazards regression,, in Handbook of Statistics in Clinical Oncology, ed. J. Crowley, Marcel Dekker Inc., pp. 397-409.

Pepe, M. S. (2003), The Statistical Evaluation of Medical Tests for Classification and Prediction Oxford University Press.

Schemper, M., and Henderson, R. (2000), "Predictive accuracy and explained variation in Cox regression," Biometrics, 56(1), 249-255.

Slate, E. H., and Turnbull, B. W. (2000), "Statistical models for longitudinal biomarkers of disease onset," Statistics in Medicine, 19(4), 617-637.

Xu, R., and O'Quigley, J. (2000), "Proportional hazards estimate of the conditional survival function," Journal of the Royal Statistical Society, Series B, Methodological, 62(4), 667-680.

Zheng, Y., and Heagerty, P. (2003), "Semiparametric estimation of time-dependent ROC curves for longitudinal marker data," University of Washington Technical Report, pp. 1-25.

Zhou, X.-H., McClish, D. K., and Obuchowski, N. A. (2002), Statistical Methods in Diagnostic Medicine John Wiley \& Sons. 
Table 1: Simulation results for estimation of I/D accuracy. Data $\left(M_{i}, \log T_{i}\right)$ were generated as bivariate normal with a correlation of $\rho=-0.7$. The sample size for each simulated data set was $N=200$. The $A U C(t)$ curve and the integrated curve, $C^{\tau}$, was estimated using: maximum likelihood assuming a bivariate normal model; Cox model which assumes proportional hazards; local maximum partial likelihood for the varying-coefficient model $\lambda(t)=\lambda_{0}(t) \exp \left[\gamma(t) M_{i}\right]$; and a local linear smooth of the scaled Schoenfeld residuals to estimate the varying-coefficient model.

\section{0\% Censoring}

MLE Cox model local MPLE residual smooth

\begin{tabular}{rrrrrrrrrr} 
log time & $A U C(t)$ & mean & (s.d.) & mean & (s.d.) & mean & (s.d.) & mean & (s.d.) \\
\hline & & & & & & & & & \\
-2.0 & 0.884 & 0.884 & $(0.018)$ & 0.743 & $(0.028)$ & 0.860 & $(0.052)$ & 0.881 & $(0.044)$ \\
-1.5 & 0.833 & 0.834 & $(0.019)$ & 0.734 & $(0.026)$ & 0.817 & $(0.033)$ & 0.829 & $(0.035)$ \\
-1.0 & 0.782 & 0.782 & $(0.019)$ & 0.725 & $(0.024)$ & 0.768 & $(0.031)$ & 0.771 & $(0.033)$ \\
-0.5 & 0.734 & 0.734 & $(0.019)$ & 0.716 & $(0.023)$ & 0.722 & $(0.032)$ & 0.720 & $(0.033)$ \\
0.0 & 0.693 & 0.693 & $(0.018)$ & 0.707 & $(0.021)$ & 0.688 & $(0.034)$ & 0.686 & $(0.034)$ \\
0.5 & 0.660 & 0.660 & $(0.016)$ & 0.700 & $(0.023)$ & 0.655 & $(0.041)$ & 0.657 & $(0.040)$ \\
1.0 & 0.634 & 0.634 & $(0.015)$ & 0.691 & $(0.028)$ & 0.633 & $(0.044)$ & 0.637 & $(0.041)$ \\
1.5 & 0.614 & 0.614 & $(0.013)$ & 0.670 & $(0.044)$ & 0.621 & $(0.064)$ & 0.622 & $(0.048)$ \\
2.0 & 0.598 & 0.598 & $(0.012)$ & 0.600 & $(0.075)$ & 0.579 & $(0.076)$ & 0.573 & $(0.060)$ \\
& & & & & & & & & \\
\hline
\end{tabular}

\section{$40 \%$ Censoring}

MLE Cox model local MPLE residual smooth

\begin{tabular}{rccccccccc}
$\log$ time & $A U C(t)$ & mean & (s.d.) & mean & (s.d.) & mean & (s.d.) & mean & (s.d.) \\
\hline-2.0 & 0.884 & 0.884 & $(0.019)$ & 0.749 & $(0.031)$ & 0.859 & $(0.054)$ & 0.875 & $(0.048)$ \\
-1.5 & 0.833 & 0.834 & $(0.021)$ & 0.742 & $(0.029)$ & 0.818 & $(0.035)$ & 0.827 & $(0.037)$ \\
-1.0 & 0.782 & 0.782 & $(0.021)$ & 0.732 & $(0.026)$ & 0.770 & $(0.035)$ & 0.772 & $(0.035)$ \\
-0.5 & 0.734 & 0.734 & $(0.020)$ & 0.722 & $(0.024)$ & 0.724 & $(0.038)$ & 0.722 & $(0.039)$ \\
0.0 & 0.693 & 0.693 & $(0.019)$ & 0.712 & $(0.024)$ & 0.689 & $(0.042)$ & 0.687 & $(0.041)$ \\
0.5 & 0.660 & 0.660 & $(0.018)$ & 0.702 & $(0.026)$ & 0.654 & $(0.045)$ & 0.655 & $(0.043)$ \\
1.0 & 0.634 & 0.635 & $(0.016)$ & 0.689 & $(0.035)$ & 0.633 & $(0.057)$ & 0.637 & $(0.048)$ \\
1.5 & 0.614 & 0.614 & $(0.015)$ & 0.653 & $(0.055)$ & 0.617 & $(0.075)$ & 0.614 & $(0.051)$ \\
2.0 & 0.598 & 0.599 & $(0.013)$ & 0.560 & $(0.073)$ & 0.555 & $(0.075)$ & 0.546 & $(0.058)$ \\
& & & & & & & & & \\
\hline
\end{tabular}


Table 2: Cox regression regression estimates for the VA lung cancer data where follow-up is truncated at 500 days. The reference category for cell type is squamous.

\begin{tabular}{lrrr}
\hline Covariate & estimate & s.e. & $\mathrm{Z}$ \\
\hline & & & \\
Treatment & -0.323 & 0.206 & -1.566 \\
Age/10 & -0.086 & 0.093 & -0.937 \\
Karnofsky score & -0.032 & 0.005 & -5.931 \\
Cell type (small) & 0.841 & 0.270 & 3.116 \\
Cell type (adeno) & 1.151 & 0.295 & 3.896 \\
Cell type (large) & 0.350 & 0.285 & 1.231 \\
\hline
\end{tabular}

Table 3: Cox regression estimates for the PBC data.

\begin{tabular}{lrrr}
\hline Covariate & estimate & s.e. & $\mathrm{Z}$ \\
\hline \multicolumn{4}{c}{ Model 1 } \\
& & \\
$\log$ (bilirubin) & 0.928 & 0.099 & 9.401 \\
$\log ($ prothrombin time) & 0.076 & 0.111 & 0.678 \\
edema & 0.967 & 0.300 & 3.221 \\
albumin & -0.961 & 0.240 & -4.001 \\
age & 0.036 & 0.009 & 4.243 \\
\hline
\end{tabular}

Model 2

\begin{tabular}{lrrr}
$\log ($ prothrombin time) & 0.148 & 0.105 & 1.41 \\
edema & 1.491 & 0.294 & 5.07 \\
albumin & -1.316 & 0.229 & -5.76 \\
age & 0.029 & 0.009 & 3.29 \\
\hline
\end{tabular}


I/D ROC curves for log-normal

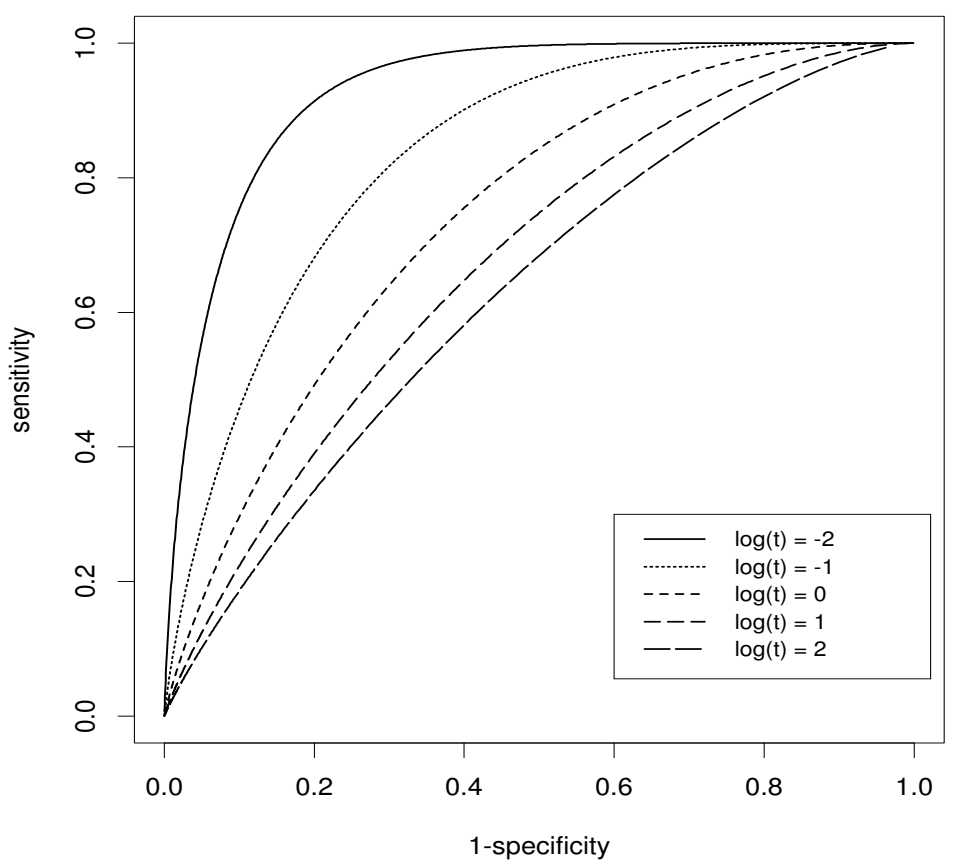

(a) Incident/Dynamic ROC curves for a scalar marker and a disease time where $\left\{M_{i}, \log \left(T_{i}\right)\right\}$ is bivariate normal with $\rho=-0.8$.

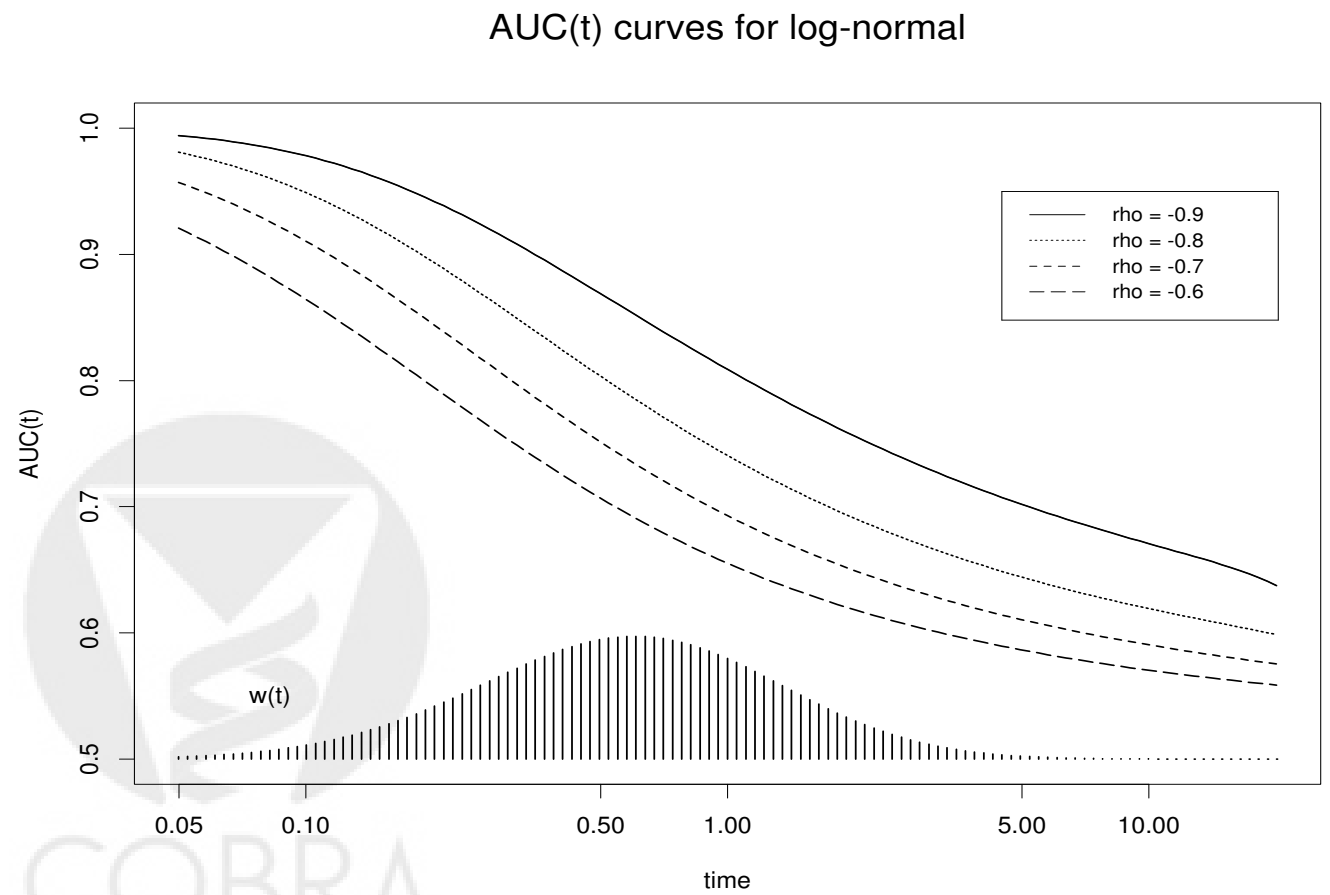

(b) Plots of $A U C(t)$ for a scalar marker and a disease time where $\left\{M_{i}, \log \left(T_{i}\right)\right\}$ is bivariate normal with $\rho$ taking the values $(-0.9,-0.8,-0.7,-0.6)$.

Figure 1: Incident/Dynamic ROC and AUC plots for a bivariate (log) normal distribution. 


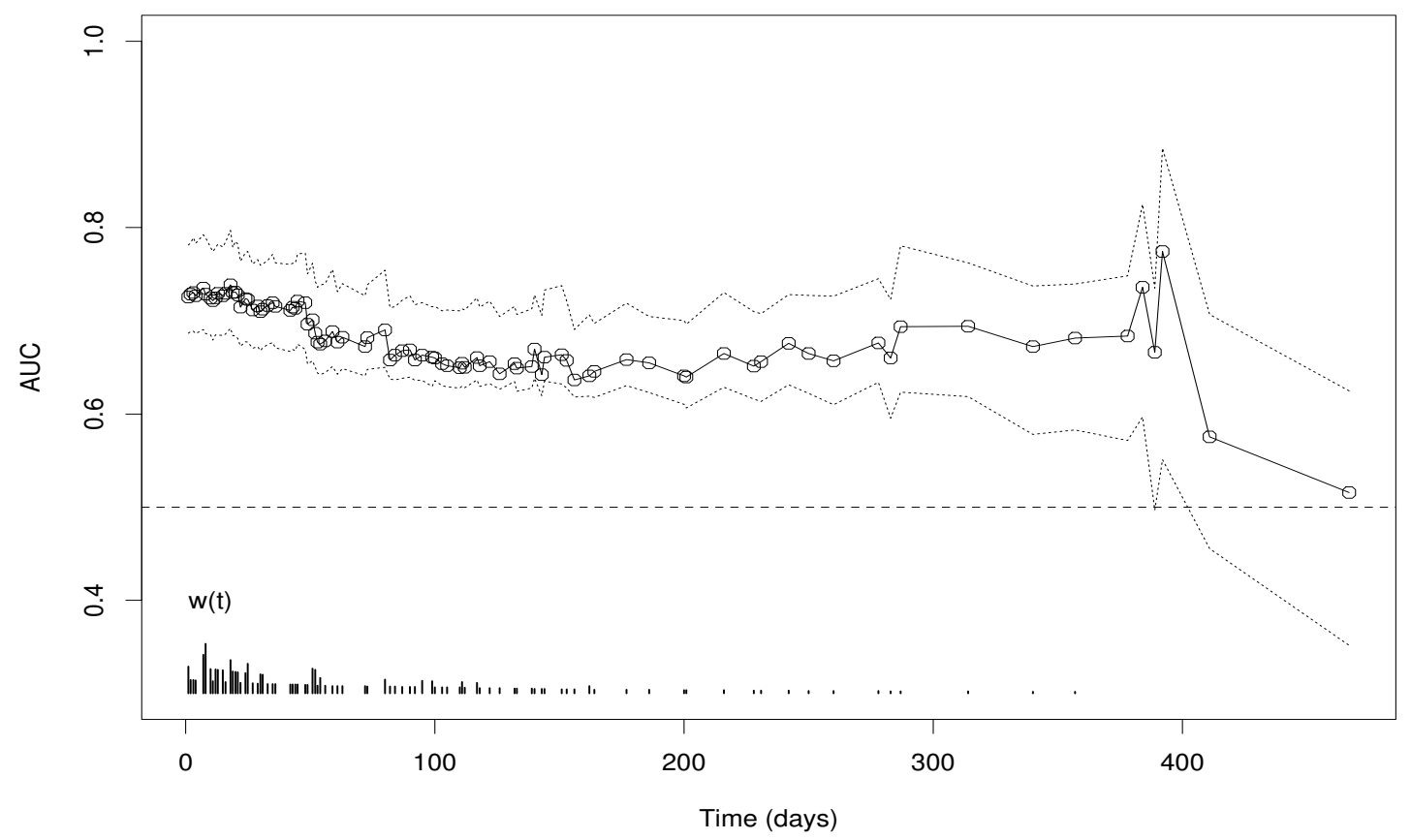

(a) Accuracy of the model score (linear predictor) under the assumption of proportional hazards. Estimates of I/D $A U C(t)$ versus time with pointwise $90 \%$ confidence intervals. Using $\tau=365$ we obtain $\widehat{C}^{\tau}=$ $\int_{0}^{\tau} \widehat{A U C}(t) \cdot \hat{w}^{\tau}(t) d t=0.713$, (s.e. $\left.=0.026\right)$.

\section{AUC based on Varying-Coefficient Cox model}

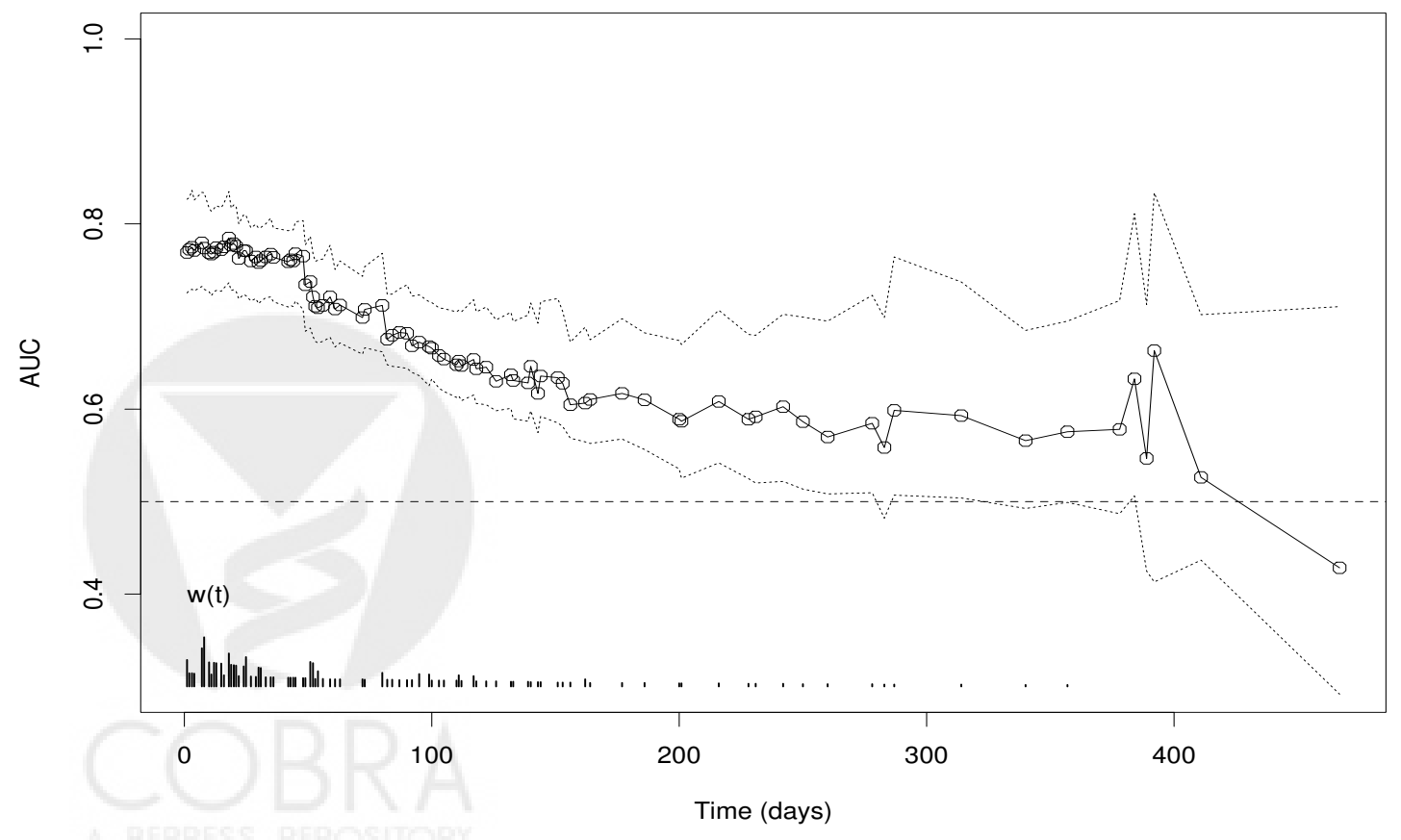

(b) Accuracy of the model score (linear predictor) based on a varying-coefficient multiplicative hazard model. Estimates of I/D $A U C(t)$ versus time with pointwise $90 \%$ confidence intervals. Using $\tau=365$ we obtain $\widehat{C}^{\tau}=\int_{0}^{\tau} \widehat{A U C}(t) \cdot \hat{w}^{\tau}(t) d t=0.738$, (s.e. $\left.=0.022\right)$.

Figure 2: Incident/Dynamic AUC plots for the VA lung cancer data. 


\section{I/D ROC curves for the model score}

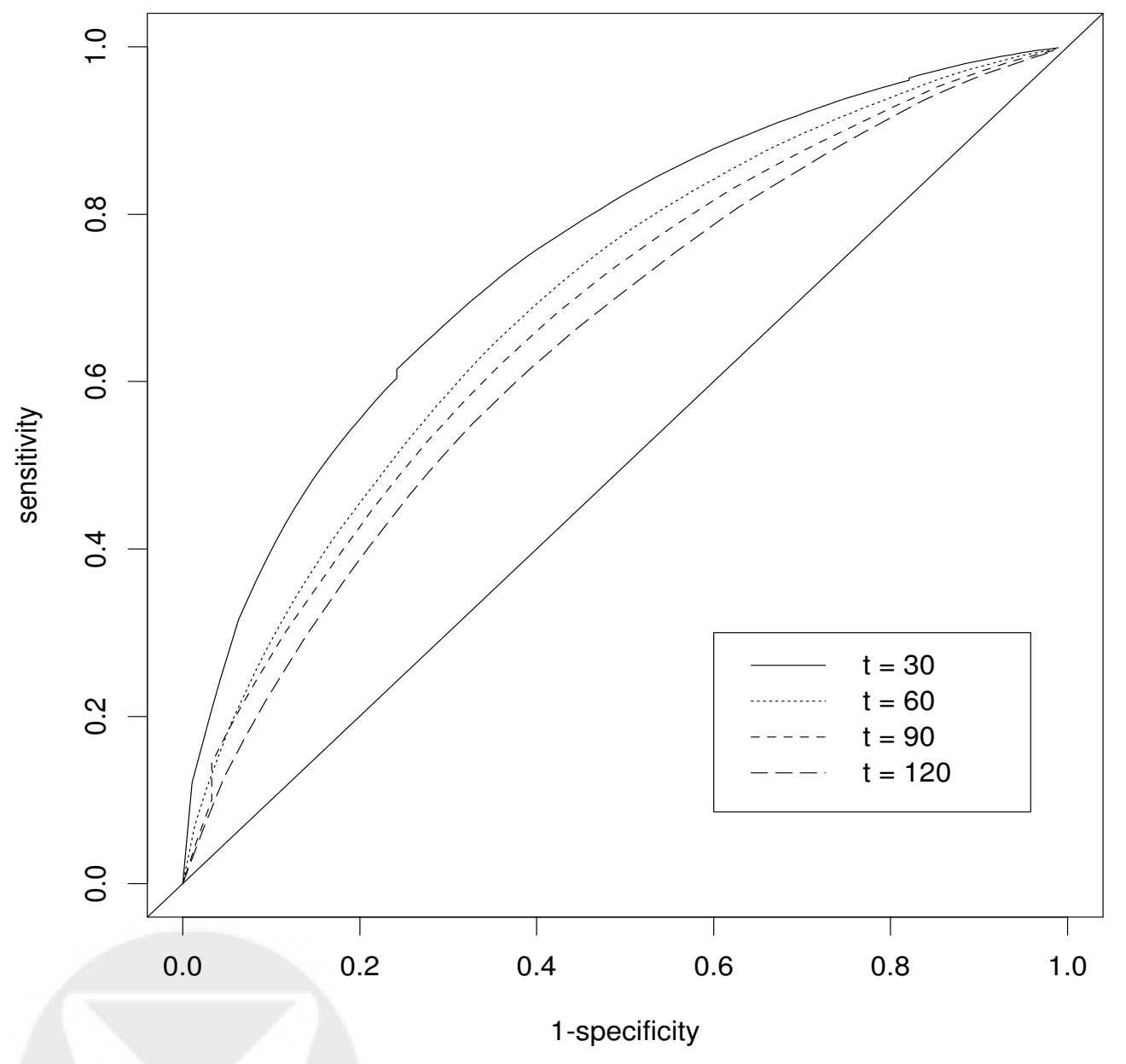

Figure 3: Incident / Dynamic ROC curves for the VA Lung Cancer data. A model score is derived using Cox regression with Karnofsky score, age, and cell type. ROC curves are estimated using a varying-coefficient Cox model with the derived model score as the single predictor. 
AUC based on Cox model

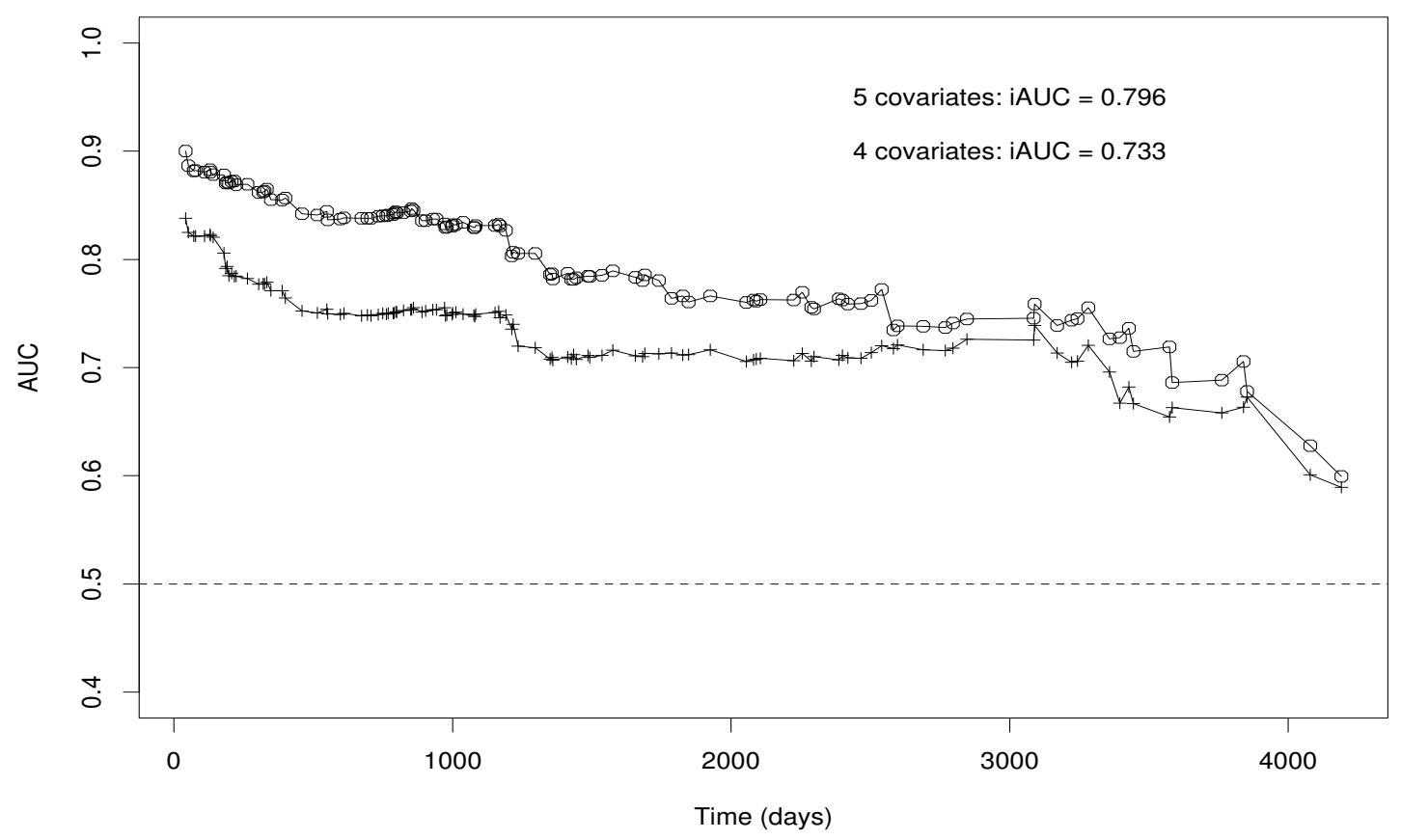

(a) Accuracy of the model score using five covariates (o) $\log$ (bilibubin), $\log$ (prothrombin), edema, albumin, age, and the model score using four covariates $(+)$ where $\log ($ bilirubin) is excluded. Lines plot the estimates of I/D $A U C(t)$ versus time under the assumption of proportional hazards.

\section{AUC based on Varying-Coefficient Cox model}

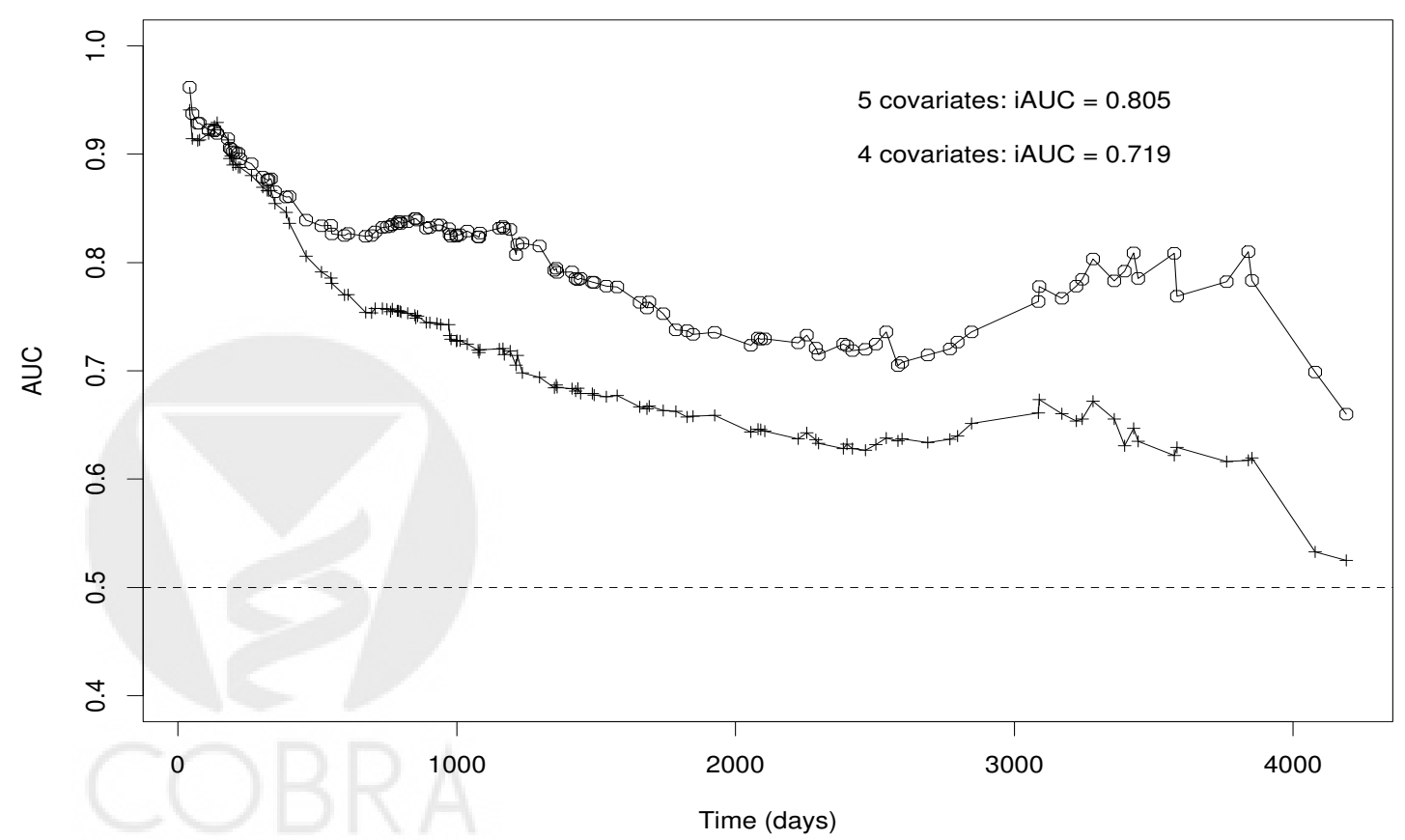

(b) Accuracy of the model score using five covariates (o) $\log$ (bilibubin), $\log$ (prothrombin), edema, albumin, age, and the model score using four covariates $(+)$ where $\log ($ bilirubin) is excluded. Estimation is based on a varying-coefficient multiplicative hazard model. Lines plot the estimates of I/D $A U C(t)$ versus time.

Figure 4: Incident/Dynamic Aß3 plots for the Mayo PBC data. 


\section{A. Appendix}

\section{A.1 Concordance as function of $A U C(t)$}

Assume independent observations $\left(M_{j}, T_{j}\right)$ and $\left(M_{k}, T_{k}\right)$, and assume that $T_{j}$ is continuous such that $P\left(T_{k}=T_{j}\right)=0$. Let $P(x)$ denote probability or density depending on the context.

$$
\begin{aligned}
& P\left[T_{j}<T_{k}\right]=\frac{1}{2} \text { (by independence) } \\
P\left[M_{j}>M_{k} \mid T_{j}<T_{k}\right]= & P\left[\left\{M_{j}>M_{k}\right\} \cap\left\{T_{j}<T_{k}\right\}\right] \times 2 \\
= & \int_{t} P\left[\left\{M_{j}>M_{k}\right\} \cap\left\{T_{j}=t\right\} \cap\left\{t<T_{k}\right\}\right] \times 2 d t \\
= & \int_{t} P\left[\left\{M_{j}>M_{k}\right\} \mid\left\{T_{j}=t\right\} \cap\left\{t<T_{k}\right\}\right] \times 2 \times P\left[\left\{T_{j}=t\right\} \cap\left\{t<T_{k}\right\}\right] d t \\
= & \int_{t} \mathrm{AUC}(t) \times 2 \times P\left[T_{j}=t\right] \times P\left[t<T_{k}\right] d t \\
= & \int_{t} \mathrm{AUC}(t) \cdot w(t) d t=E_{T}[\mathrm{AUC}(T) \times 2 \times S(T)] \\
& \quad \text { iith } w(t)=2 \cdot f(t) \cdot S(t)
\end{aligned}
$$

\section{A.2 Hazard as bridge from $P\left(M_{i}=m \mid T_{i} \geq t\right)$ to $P\left(M_{i}=m \mid T_{i}=t\right)$}

Let $P(x)$ denote probability or density depending on the context and specific assumptions. For either continuous or discrete survival times the conditional hazard can be defined as:

$$
\lambda\left(t \mid M_{i}=m\right)=P\left(T_{i}=t \mid M_{i}=m\right) / P\left(T_{i} \geq t \mid M_{i}=m\right) .
$$

Let $P(m)$ denote the marginal density or distribution of the marker $M$. Following Xu and O'Quigley (2000) we obtain the following general relationship:

$$
\begin{aligned}
P\left(M_{i}=m \mid T_{i}=t\right) & =P\left(T_{i}=t \mid M_{i}=m\right) \cdot P\left(M_{i}=m\right) / P\left(T_{i}=t\right) \\
& =\lambda\left(t \mid M_{i}=m\right) \cdot P\left(T_{i} \geq t \mid M_{i}=m\right) \cdot P\left(M_{i}=m\right) / P\left(T_{i}=t\right) \\
& =\lambda\left(t \mid M_{i}=m\right) \cdot P\left(M_{i}=m \mid T_{i} \geq t\right) \cdot P\left(T_{i} \geq t\right) / P\left(T_{i}=t\right) \\
P\left(M_{i}=m \mid T_{i}=t\right) & \propto \lambda\left(t \mid M_{i}=m\right) \cdot P\left(M_{i}=m \mid T_{i} \geq t\right) .
\end{aligned}
$$

